\title{
Norois
}

Environnement, aménagement, société

$214 \mid 2010 / 1$

Le Maroc en mutation

\section{L'eau dans le bassin du Souss : concurrences et désorganisation des systèmes paysans}

Water in the Souss basin: competition and disorganization of the rural systems

\section{Mohamed Boujnikh et André Humbert}

\section{(2) OpenEdition}

1 Journals

\section{Édition électronique}

URL : https://journals.openedition.org/norois/3178

DOI : 10.4000/norois.3178

ISBN : 978-2-7535-1562-8

ISSN : $1760-8546$

Éditeur

Presses universitaires de Rennes

Édition imprimée

Date de publication : 20 mai 2010

Pagination : 113-126

ISBN : 978-2-7535-1121-7

ISSN : 0029-182X

\section{Référence électronique}

Mohamed Boujnikh et André Humbert, «L'eau dans le bassin du Souss : concurrences et

désorganisation des systèmes paysans », Norois [En ligne], 214 | 2010/1, mis en ligne le 01 juin 2012, consulté le 13 janvier 2022. URL : http://journals.openedition.org/norois/3178 ; DOI : https://doi.org/ $10.4000 /$ norois. 3178

(c) Tous droits réservés 


\title{
L'EAu DANS LE BASSin du Souss :
}

\section{CONCURRENCES ET DÉSORGANISATION DES SYSTÈMES PAYSANS}

\author{
Mohamed Boujnikh, André Humbert \\ Centre d'Études et de Recherches sur les Paysages (CERPA) \\ (université Nancy 2), \\ 3, place Godefroy-de-Bouillon - 54015 NANCY cedex \\ m_boujnikh@hotmail.com,andrehumbert@aol.com
}

\section{RÉSUMÉ}

La plaine du Souss est aujourd'hui la première région marocaine pour la production des agrumes (plus de $40 \%$ ) et des primeurs (plus de $60 \%$ ). C'est au cour de la plaine que le front pionnier, fondé sur le pompage des aquifères, a débuté vraiment après la Seconde Guerre mondiale. Il a progressé ensuite vers l'aval de la dépression avec surtout du marâेchage. La phase la plus récente a gagné le Souss amont, à l'est de Taroudant. Le succès économique de cette agriculture véritablement minière a une contrepartie regrettable avec la disparition des systèmes traditionnels d'irrigation qui utilisaient la partie supérieure des nappes aujourd'hui en cours d'épuisement. Mais au-delà de la menace de disparition d'une vieille civilisation de l'eau, c'est la durabilité des systèmes modernes qui est en question.

MoTS CLÉ : agriculture minière - agrumiculture-aquifère - culture sous serres - développement durable - dir - galerie drainante (qanat) - irrigation traditionnelle

\footnotetext{
ABSTRACT

Water in the Souss basin: competition and disorganization of the rural systems

The plain of the Souss is today the first moroccan area for the production of citrus fruits (more than 40\%) and early products (more than 60\%). It is in the middle of the plain that the pioneer core, founded on the pumping of the aquifers, really began after the Second World War. It progressed then towards the downstream of the depression with, especially, market gardening. The most recent stage gained upper Souss, in the east of Taroudant. The economic success of this truly mining agriculture has a regrettable counterpart with the disappearance of the traditional systems of irrigation, which used the higher part of the underground water reserves, today in the course of exhaustion. But beyond the threat of disappearance of an old civilization of water, it is the sustainability of the modern systems which is in question.
}

KEY WORDS : mining agriculture - citrus fruits farming - aquifer - greenhouses farming - sustainable development - dir - draining off tunnel (qanat) - traditional irrigation 
Les concurrences autour de l'eau, les conflits potentiels ou ceux qui éclatent réellement constituent des phénomènes planétaires alimentant le débat sur le développement durable (Galland, 2008). Les régions arides ou subarides ne sont pas les seules à êtres concernées mais les problèmes auxquels elles sont confrontées y sont plus aigus (et plus anciens) que dans les espaces mieux pourvus de la planète (Mutin, 2000; Margat, 2008). En dépit d'une pénurie chronique, des sociétés paysannes stables y ont fonctionné pendant des siècles (des millénaires parfois) sinon de façon paisible, du moins dans un certain équilibre avec les ressources hydriques disponibles (Pérennès, 1993). Le Maghreb est un de ces espaces de vieille civilisation rurale fondée en grande partie sur l'irrigation. Au Maroc où les montagnes, étendues et élevées sont de riches châteaux d'eau, les communautés paysannes ont sécrété des paysages qui expriment les bienfaits de l'eau. Ces terroirs généreux ont cependant dû supporter une pression démographique grandissante et sont entrés au $\mathrm{Xx}^{\mathrm{e}}$ siècle en concurrence avec de nouveaux utilisateurs de la terre et de l'eau. Les puissances coloniales - la France mais aussi l'Espagne dans le Nord - ont voulu mobiliser de nouvelles ressources avec de nouveaux moyens technologiques (Despois et Raynal, 1967). Dès avant l'indépendance, le Maroc est pourvu d'un certain nombre d'ouvrages hydrauliques permettant une extension considérables des terres irriguées. C'est surtout dans le nord (plaine du Gharb) et le centre (Tadla) qu'a été amorcée la révolution hydraulique. Au sud du Haut-Atlas, les possibilités sont bien moindres et aucun équipement majeur n'est venu modifier sérieusement la situation. Au cours des quarante dernières années, une économie agricole très dynamique, toute entière fondée sur l'exploitation des ressources hydriques, s'est installée progressivement. Quelles mutations des paysages et des systèmes agraires anciens cette modernisation de l'agriculture a-t-elle entraînées?

\section{La plaine du Souss : la plus méridionale des grandes plaines atlantiques marocaines}

Mieux délimitée que les grandes plaines atlantiques de la Chaouia ou des Doukkala, plus symétrique aussi dans sa topographie que le Gharb ou le Haouz, la plaine du Souss, ou le Souss, comme l'on dit plus volontiers, est parfaitement dessinée entre le Haut-Atlas et l'Anti-Atlas. Cette dépression tectonique triangulaire s'étend sur plus de $150 \mathrm{~km}$ d'ouest en est venant mourir en pointe, à l'amont, contre le massif du Siroua. D'une quinzaine de kilomètres seulement à l'amont, sa largeur dépasse $50 \mathrm{~km}$ sur le littoral si l'on fait abstraction du décrochement au sud que forme la plaine de Tiznit (fig. 1).

La plaine du Souss offre aujourd'hui une grande variété de paysages organisés en grandes bandes parallèles à son axe fluvial. Une première bande particulièrement anthropisée foisonne de gros villages et de bourgades noyés dans des faciès culturaux contrastés qui s'opposent aussi bien par leurs teintes que par leur texture. Dans ce large ruban axial, des terroirs de plaine à la structure parcellaire anarchique cohabitent, en effet, avec leurs douar $(\mathrm{s})^{1}$ et le vaste cercle des orangeraies aux pièces inégales par la taille, au dessin géométrique, et parsemées de quelques grands bâtiments isolés (photo 1 - planche II). Cet ordonnancement est surtout celui du centre de la plaine autour d'Ouled Teima. Ailleurs, à l'aval et à l'amont, les étendues d'orangeraies sont fréquemment trouées de grands blocs de serres recouvertes de plastique et, parfois, de grands cercles d'irrigation par rampes pivotantes. Enfin, quelques damiers parfaitement rigoureux, associés à des villages au plan systématique quadrillé, semblent vouloir imposer un peu d’ordre administré.

Ce vivant ruban axial est encadré de deux bandeaux qui contrastent avec les terroirs précédents par leur vacuité. Il s'agit des deux longs glacis qui s'élèvent en pente douce vers les fronts montagneux, livrés à une agriculture pluviale de hasards et parsemés de maigres arganiers.

1. Les définitions des termes vernaculaires utilisés dans cet article sont regroupées dans le lexique à la fin de ce numéro spécial Maroc de la revue Norois. 


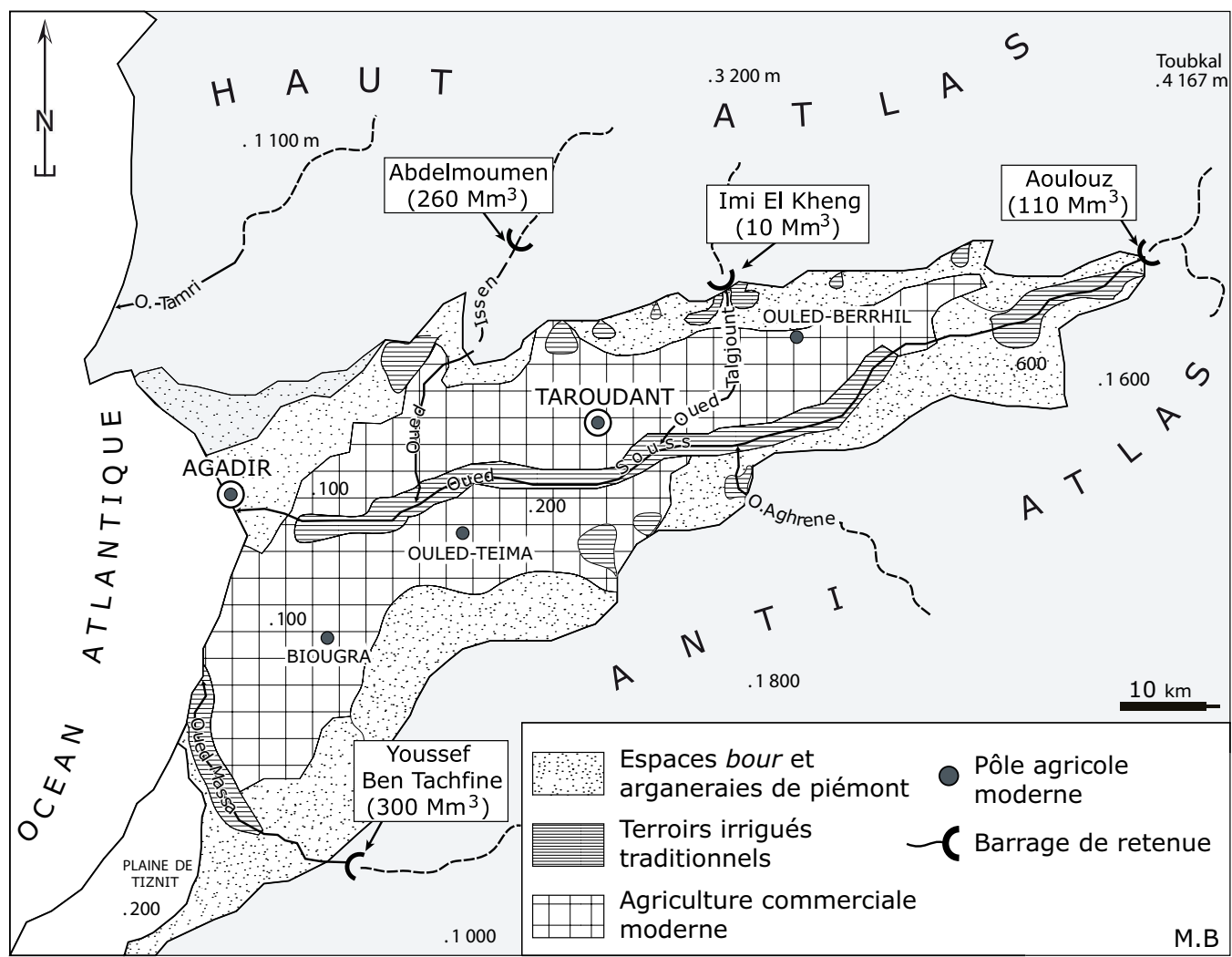

Figure 1 : Carte de localisation et paysages agraires de la vallée du Souss (réalisation M. Boujnikh) Location map and agrarian landscapes of the Souss valley

La vie réapparaît au contact de la montagne avec des villages installés au débouchés des vallées, en bordures des foum (s) qui livrent l'eau de la montagne à des terroirs verdoyants qui s'étalent sur les cônes de déjection et finissent par s'évanouir dans la steppe. Ce chapelet de foyers de vie est le dir, le « poitrail » que la montagne dresse au-dessus de la plaine.

\section{Une plaine conquise tardivement par l'hydraulique moderne}

Les Européens se sont intéressés très tôt aux terres de culture au Maroc. Dès avant la Première Guerre mondiale, ils en possédaient 70000 ha et, au début des années 1930, ils étaient à la tête de grandes exploitations qui couvraient sans doute plus de 600000 ha (Lugan, 2000). Mais ces terres étaient pour la plupart dans les «bons pays » du nord, dans la plaine du Saïs (Fès), du Rharb ou de la Chaouia, dans le "Maroc utile », et non pas sur ses marges arides de peu d'intérêt économique. Les programmes de grande hydraulique beaucoup plus tardifs qu'en Algérie ont d'abord été appliqués aux territoires du nord, particulièrement au Gharb, afin de contrôler plus efficacement les crues redoutables dans le bassin de l'oued Sebou (Martin et al., 1970). Il est vrai que, dès les années 1930, un barrage a été établi sur le piémont du Haut-Atlas, près de Marrakech pour améliorer l'exploitation de la partie de la plaine du Haouz proche de Marrakech (barrage de Lala Takerkoust et périmètre du N'fis). C'est à la même époque que sont engagées les études pour un équipement du bassin de l'Oum er Rbia réalisé progressivement à partir de 1949, 
Mohamed Boujnikh, André Humbert

et qui a permis d'étendre considérablement les périmètres irrigués modernes dans les plaines du Tadla et des Doukkala (Troin, 1985).

Aucun ouvrage significatif n'a été réalisé au profit de l'agriculture du Souss avant les années 1970. Il est incontestable que le potentiel hydraulique n'est pas celui des montagnes bien arrosées du Nord ou du Moyen-Atlas. La plaine est cependant dominée par le Haut-Atlas occidental enneigé une partie de l'année et relativement bien exposé aux perturbations du front polaire qui atteignent cette latitude. Ce n'est pourtant pas là qu'a été réalisé le premier ouvrage, mais sur la bordure de la partie sud-occidentale de la plaine, sur le principal organe fluvial issu de l'Anti-Atlas, l'oued Massa. La mise en service de ce premier barrage soussi (Youssef Ben Tachfine), destiné à la création d'un périmètre irrigué exemplaire, a eu lieu en 1972 seulement. Il a une capacité modeste de $300 \mathrm{hm}^{3}$ correspondant approximativement à deux ans d'apport pluvial moyen interannuel. Les vallées du Haut-Atlas ont été équipées plus tard encore avec des ouvrages plus modestes. Le principal (Abdelmoumen) a été aménagé sur l'oued Issen, au nord-est d'Agadir. Sa mise en eau date de 1981 et sa capacité n'est que de $216 \mathrm{hm}^{3}$. Il a néanmoins permis d'améliorer un secteur d'irrigation traditionnelle situé entre le piémont de l'Atlas et l'oued Souss, et aussi de pourvoir en eaux de surface toute une partie de la zone assoiffée des vergers d'agrumes de la rive gauche du Souss autour d'Ouled Teima.

La mise en eau tardive de ces ouvrages, somme toute bien modestes, ne signifie pas que l'on ait dû attendre les années 1970 ou 1980 pour observer une mise en valeur de la plaine du Souss par l'hydraulique moderne. En effet, la mobilisation des eaux de surface est intervenue ici bien longtemps après qu'eut été entreprise une colonisation agricole moderne, grâce aux ressources souterraines contenues dans les nappes superficielles et surtout dans les aquifères profonds de l'énorme masse sédimentaire du remblaiement de la dépression.

Les Français ont amorcé cette évolution dans le centre de la plaine, c'est-à-dire autour d'Ouled Teima, dès la fin des années 1930, essentiellement avec des agrumes et des cultures maraîchères, après que des études hydrogéologiques eurent montré la richesse des aquifères multiples des formations néogènes et quaternaires (Dijon, 1969). Le progrès de cette mise en valeur est demeuré cependant très modeste avant la guerre. En 1940, seulement une centaine d'hectares était exploitée en orangers et maraîchage, autour d’Ouled Teïma. (Hnaka, 1995; Popp, 1984). Cette agriculture irriguée commerciale, fondée sur le pompage dans les nappes, s'est épanouie après la guerre, surtout après 1965 lorsqu'un effort pour favoriser la commercialisation des productions et faciliter les équipements nécessaires a été entrepris par les pouvoirs publics, notamment avec la création de l'Office de Commercialisation et d'Exportation (OCE) et de la Caisse Nationale de Crédit Agricole (Hnaka, 1995). Les structures foncières associées à cette nouvelle agriculture dynamique sont plus variées qu'on ne le dit généralement. Il y a certes de grandes propriétés dont les titulaires vivent à Casablanca ou à Rabat, mais il y a aussi un nombre considérable de petites tenures entre les mains d'une paysannerie locale qui s'est lancée dans l'aventure, en utilisant parfois de l'argent gagné en Europe. Cette hétérogénéité des structures agraires apparaît évidente à l'observation des photos aériennes (photo 1 - planche II). Les puits de débit variable se sont multipliés en même temps que les surfaces équipées explosaient littéralement. La première moitié de la décennie de 1970 a été particulièrement dynamique. En 1970, il y avait environ 10600 ha d'agrumes dans la vallée du Souss. Six ans plus tard il y en avait 19000 ha, soit près du double (Popp, 1984). Au début du $\mathrm{XXI}^{\mathrm{e}}$ siècle, les superficies totales dépassent 27000 ha dont 25000 sont concentrés dans la seule province de Taroudant (Berriane, 2002). Les progrès récents du front pionnier sur le glacis de piémont du Haut-Atlas sont souvent réalisés sur les terres des communautés (jmâ̂) qui les louent, ou même les vendent, aux « entrepreneurs » parfois étrangers à la région. Dès les années 1970, les prélèvements dans les nappes étaient supérieurs à leur recharge et l'on pouvait alors déjà observer un rabattement inquiétant du niveau piézométrique. Il était d’ores et déjà passé de 10-20 m à 30-40 m de profondeur dans les années 1960 (Dijon, 1969; Popp, 1984, 1986). La situation est alors devenue réellement préoccupante et les pouvoirs publics ont considéré qu'il était urgent de 
mobiliser les eaux de surface de l'Atlas occidental pour étendre l'irrigation sur les piémonts et venir conforter celle des terres à agrumes de la région d'Ouled Teima (Baroud et El Arabi, 2005).

Vers les extrémités aval et amont de la plaine, et dans un premier temps, les nappes ont été moins sollicitées, même si une couronne maraîchère s'était développée autour d'Agadir. Cette dernière a ensuite progressé vers le sud pour envahir les terres des Chtouka autour de Biougra où s'est épanouie la culture sous serres des tomates, des haricots verts, des cucurbitacées diverses et ...des bananiers. C'est à l'amont, à l'est de Taroudant, que la vague est arrivée le plus tard, du moins sous la forme dominante d'une exploitation capitaliste des terres et de l'eau. En effet, dans les années 1970, l'État marocain a décidé d'utiliser une partie des vastes terrains de statut domanial (maghzen) ou collectif (jmâa) pour réaliser une réforme agraire au profit d'une petite paysannerie. Cette réforme fut fondée sur l'exploitation de l'eau des nappes par le biais de systèmes très modernes. Ce sont plus de 6000 ha, répartis en une dizaine de périmètres, qui ont été progressivement équipés et distribués en lots de 5 ha environ à des paysans réunis en coopératives (Popp, 1984). Ces périmètres nouveaux sont bien reconnaissables à leur géométrie rigoureuse (photo 2 - planche III). S'ils ne sont pas destinés à la culture des agrumes, ils prélèvent néanmoins des quantités d'eau considérables dans les aquifères de la partie amont de la plaine.

Mais au cours des 10 ou 15 dernières années, le front conquérant d'une agriculture capitaliste, que certains considèrent maintenant comme prédatrice, s'est avancé vers l'est et sur le vaste glacis qui monte lentement vers le Haut-Atlas, dans le secteur des Ouled Berrhil. Le nouveau paysage est plus diversifié que celui du centre de la dépression. Si les agrumes sont présents sous la forme de parcelles géantes, ils sont mêlés à des blocs parfois considérables de serres sous lesquelles on trouve le cortège habituel, mais également du raisin de table (photo 3 - planche IV). L'ancien village d'Ouled Berrhil lui-même est devenu le troisième pôle du Souss rural pour son dynamisme, après Ouled Teima et Biougra. Enfin, dans certains secteurs, parfois au milieu des reliques d'arganeraies, l'eau pompée est distribuée par des rampes pivotantes pour irriguer du maïs ou de la luzerne!

\section{Les effets de l'hydraulique moderne sur les systèmes paysans traditionnels}

Si l'agriculture moderne du Souss s'est largement établie sur les terres de céréaliculture pluviale (bour) des glacis ou sur les sables de la plaine des Chtouka, les terroirs paysans irrigués étaient présents depuis bien longtemps au long du Souss et sur le dir des deux Atlas. Les vieux terroirs des terrasses alluviales de l'oued Souss se reconnaissent facilement à leur parcellaire anarchique dont les mailles sont fréquemment soulignées par des rangées d'oliviers. Certains sont encore bien individualisés dans quelques secteurs où la vague des agrumes n'est pas parvenue, notamment à l'extrémité orientale de la dépression, même si le contenu cultural a été modifié par la « contagion » agrumicole ou maraîchère (photo 4 - planche $\mathbf{V}$ ). Ce bled seguia, qui a nourri des générations de paysans et alimenté les souk(s), utilisait certes les eaux de crue du Souss ou de ses affluents mais pouvait aussi compter sur une alimentation plus régulière d'artefacts hydrauliques, souvent collectifs, qui prélevaient l'eau des aquifères enfouis dans les différents niveaux de terrasses ou dans les alluvions du lit lui-même. Les plus spectaculaires - et les plus efficaces - de ces dispositifs sont les galeries de captage - ou de drainage - creusées dans la masse des alluvions sur des longueurs qui peuvent dépasser plusieurs kilomètres. Ces dispositifs qu'il est impossible de dater sont sans doute apparus dans la région en même temps que l'agriculture irriguée (Balland, 1992). De beaux exemples existent sur les deux rives du Souss amont mais, dans cette même partie de la plaine les plus nombreux forment des faisceaux serrés qui drainent - ou drainaient - les aquifères du piémont (fig. 3). Elles sont parfaitement repérables d'avion grâce au chapelet de puits qui jalonnent leur parcours en surface, surmontés de leur cratère de déblais. Ces galeries finissent par émerger pour se prolonger par un canal qui distribue l'eau par gravité dans l'ensemble du terroir. Euvre communautaire, la galerie de captage, que l'on appelle khettara dans tout le Sud marocain, impose une indiscutable cohésion au groupe humain qui en vit : cohésion nécessaire pour le creusement 
Mohamed Boujnikh, André Humbert

et l'entretien du dispositif, mais cohésion aussi pour la gestion de l'eau dont la répartition obéit à des règles strictes (Humbert, 2006).

Les khettara(s) sont beaucoup plus rares dans le centre et surtout l'ouest de la plaine où les aquifères sont pourtant abondants. C'est que la structure des nappes et la topographie du terrain ne facilitent pas leur creusement et surtout l'émergence des tunnels à la surface. Chez les Chtouka, dans la partie ouest de la plaine, le système le mieux adapté est celui du puits à traction animale, de quelques dizaines de mètres de profondeur et d'une efficacité limitée mais qui relève de la gestion individuelle des familles (photos $5 \mathrm{a}$ et $\mathrm{b}$ - planche VI). Ces puits, appelés arghrour(s), ou naora(s) dans certains secteurs, formaient de véritables nébuleuses autour des villages où ils ont donné naissance à des parcellaires au maillage en relation avec la capacité d'arrosage de chaque

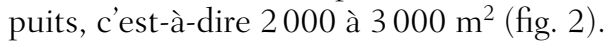

C'est finalement dans les terroirs du dir que le système de récupération de l'eau d'irrigation est le plus simple. Le plus souvent, il s'agit seulement de dériver l'eau des cours d'eau, parfois pérennes, qui sortent de la montagne. Un simple barrage de dérivation (ced ou ouggoug en berbère) alimente un canal (la seguia) dont les ramifications et les capillaires irriguent l'ensemble du terroir. A l'écart des «turbulences » de la plaine, ces terroirs sont des conservatoires de formes et de pratiques paysannes dont la survivance est favorisée par la désertion des jeunes attirés par les possibilités d'emploi dans les fermes et les agglomérations de la vallée (Humbert, 2006).

On le voit donc, deux mondes se côtoient ou se mêlent dans le Souss, deux sociétés rurales qui s'opposent par leurs ambitions, par leur conception de l'agriculture, par leurs pratiques culturales, par les paysages qu'elles engendrent et par leur mode de vie. Avec la cohabitation et les phénomènes d'imitation, les différences peuvent s'estomper. La fusion est relativement avancée dans la gouttière axiale où les fellah(s) se sont efforcés d'imiter les « entrepreneurs » des grandes fermes en modernisant leurs pratiques et en introduisant les cultures commerciales dans les vieilles structures parcellaires. Les différences restent toujours beaucoup plus marquées dans les terroirs éloignés de l'oued Souss.

Mais la cohabitation entre les deux systèmes ne saurait se prolonger paisiblement bien longtemps. En effet, ils sont concurrents pour l'espace et encore davantage pour l'eau. Les fermes d'agrumes et les serres ont besoin de terres qu'elles trouvent parfois sur les étendues collectives (jmaa) à la périphérie des terroirs villageois, mais qu'elles se procurent aussi sur les vieilles terres d'irrigation abandonnées par les fellah(s). La concurrence autour de l'eau donne lieu à une opposition totalement déséquilibrée au profit de l'agriculture moderne et, à l'intérieur même de cette catégorie, aux structures les plus puissantes dont les surfaces dépassent parfois 500 ha d'agrumes et/ou de serres. Le rabattement des aquifères dans le centre de la plaine s'est fait sentir très tôt, dès la fin des années 1960. Et ce sont les petits propriétaires qui s'étaient lancés dans l'aventure qui en ont été les premières victimes car ils n'avaient pas les ressources financières nécessaires à la " poursuite de l'eau » dans le sous-sol (Popp, 1984). Les vieux bled(s) seguia qui fonctionnent avec un ancestral droit à l'eau peuvent sembler mieux protégés. Les terroirs des $\operatorname{dir}(\mathrm{s})$ qui utilisent souvent des eaux de surface subissent une concurrence relativement modérée car l'agriculture moderne n'a pas pénétré dans les vallées des montagnes. Si ce n'est que les périmètres de la plaine doivent maintenant faire appel aux eaux de la montagne que l'on a partiellement stockées dans des lacs-réservoirs. Les communautés situées à l'aval de ces retenues n’ont pas toujours été victimes de la modification des écoulements car elles ont pu, le plus souvent, obtenir le maintien de leurs droits d'eau et parfois même bénéficier d'une extension et d'une modernisation de leurs terroirs. Ce fut le cas du dir du Haut-Atlas au débouché de l'oued Issen sur lequel a été établi le barrage Abdelmoumen (Baroud et El Arabi, 2005). Au long de l'Anti-Atlas central, de beaux terroirs étalent encore leurs oliveraies sur les cônes du piémont mais les fellah(s) se plaignent parfois du tarissement de certaines sources ou résurgences, phénomènes qu'ils attribuent facilement à une péjoration climatique mais qui pourrait, en réalité, être la manifestation lointaine du rabattement de l'aquifère de la plaine.

Les effets du surpompage sur les artefacts familiaux de la plaine des Chtouka ont été particulièrement spectaculaires. On peut observer aujourd'hui un véritable cimetière de puits à dlou [arghour(s)], 


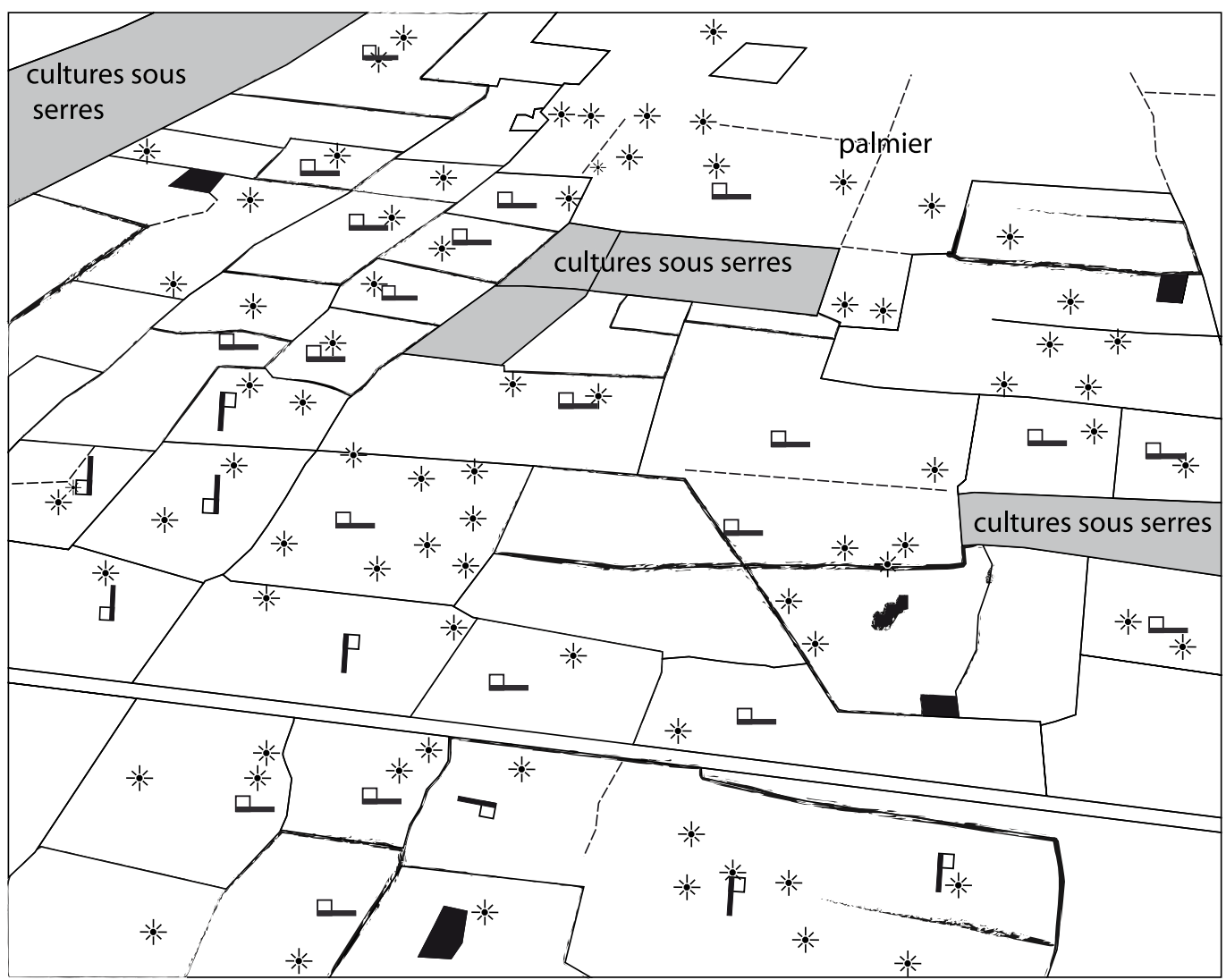

habitation

Figure 2 : Ancien terroir irrigué par arghrour(s) et abandonné à la suite du rabattement de l'aquifère au sud d'Agadir (commune de Sidi Bibi) (réalisation de M. Boujnikh à partir d'une photographie de A. Humbert, 2003)

Ce croquis, réalisé à partir d'une photographie aérienne oblique, montre bien que ces terroirs à puits familiaux étaient organisés selon un module de surface parcellaire correspondent à la capacité d'irrigation d'un artefact à traction animale, soit environ 25 ares. Tous les puits ont été stérilisés par le rabattement de la nappe mais on les repère encore facilement avec leurs superstructures en ruine et leur piste de halage dont la longueur correspondait à la profondeur atteinte. De l'ancien paysage cultural ne subsistent que des palmiers dispersés dans les champs et qui sont en train de dépérir. Les terres délaissées ont commencé à être réinvesties par des « entrepreneurs " de cultures sous serres qui s'efforcent de rassembler quelques parcelles contigües pour atteindre la taille nécessaire à l'établissement des grandes nefs de plastique.

Old irrigated fields by arghrour(s) deserted under effects of water level dropping down, south of Agadir (Sidi Bibi district)

This sketch, realized from an oblique air photograph, well shows that this area with family wells was organized according to a module of compartmental surface corresponding to the capacity of irrigation of an artifact with animal haulage, that is to say approximately (2,500 square meters). All the wells were sterilized by the lowering of the water table but one still easily locates them with their superstructures in ruin and their track of haulage of which the length corresponded to the depth reached. Of the old farming landscape remain only the palm trees dispersed in the fields and which are withering. The deserted grounds started to be reinvested by "contractors" of cultivation under greenhouses which endeavour to gather some contiguous plots to reach the size necessary to the establishment of the great plastic naves. 


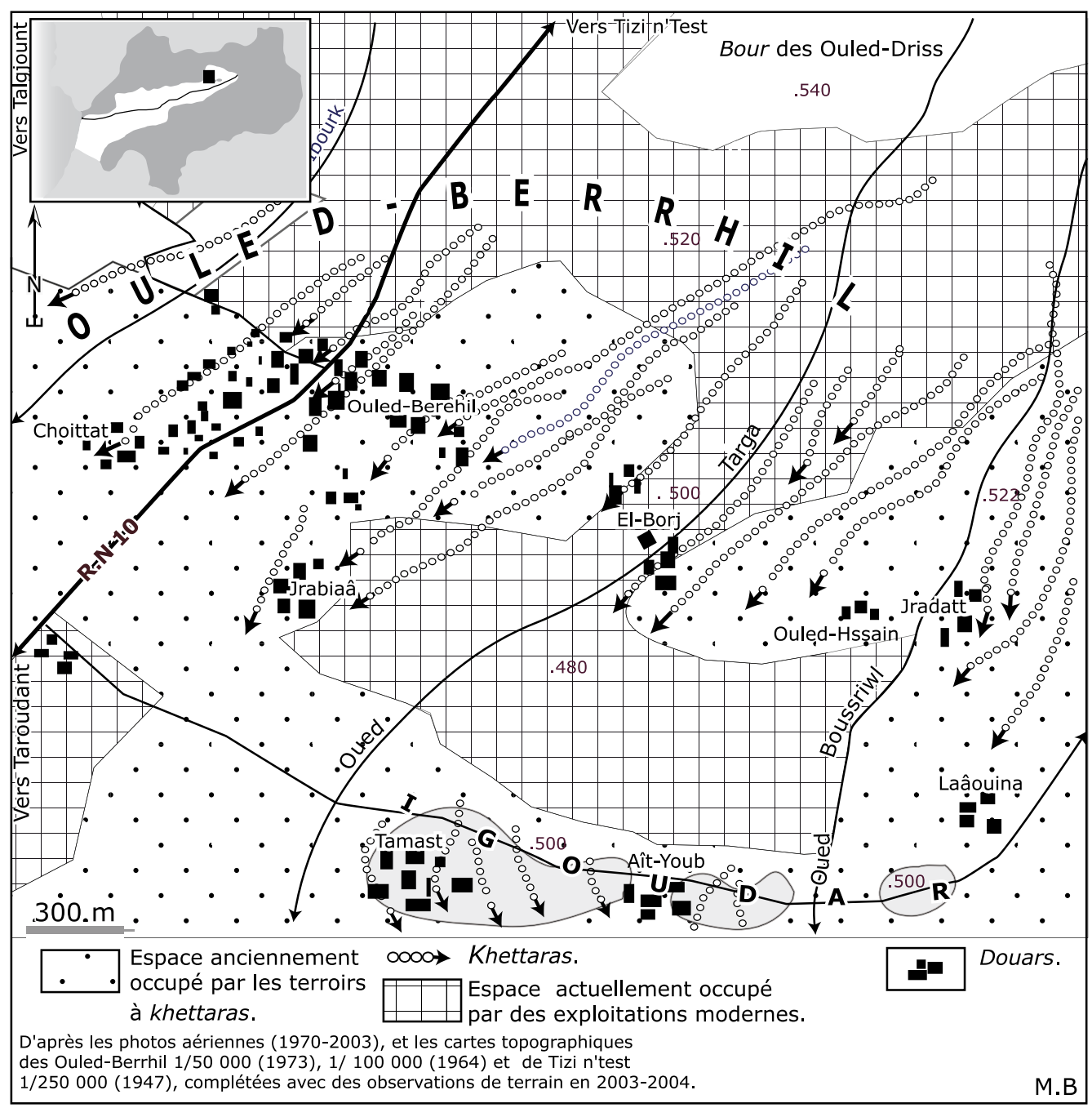

Figure 3 : Les khettara(s) mortes des Ouled Berrhil (Extrait de Boujnikh, 2008)

Autour d'Ouled Berrhil, le faisceau de khettara(s) est particulièrement dense : les plus longues mesurent presque $2 \mathrm{~km}$. C'est dans leur aire de drainage que les grandes exploitations modernes se sont installées, dans la partie inférieure du glacis du Haut-Atlas, avec des centaines de puits profonds. Pas une seule galerie n'a survécu!

The dead khettara(s) in the Ouled Berrhil area

Around Ouled Berrhil, the network of khettara(s) is particularly dense; the longest measure almost $2 \mathrm{~km}$. It is in their drainage area that the modern large farms settled, in the lower part of the glacis of the High-Atlas, with hundreds of deep wells. Not a single gallery did survive!

dans la commune de Sidi-Bibi (fig. 2 et photo $5 c$ - planche VI). Le phénomène a été rapide en dépit de la fourniture d'eau à la zone par le barrage Youssef ben Tachfine. Les eaux retenues sont en effet insuffisantes à satisfaire les bénéficiaires des lots du périmètre qui ont effectué des forages atteignant maintenant plus de $100 \mathrm{~m}$. Le rabattement est d'autant plus sévère que tout autour du périmètre officiel se sont implantées de grandes fermes de serres (secteur de Biougra).

Tout aussi spectaculaire est la mort des réseaux de captage du Souss amont. Ils ont un temps été préservés par le retard pris par l'invasion de l'agriculture commerciale. Dès que les grandes 


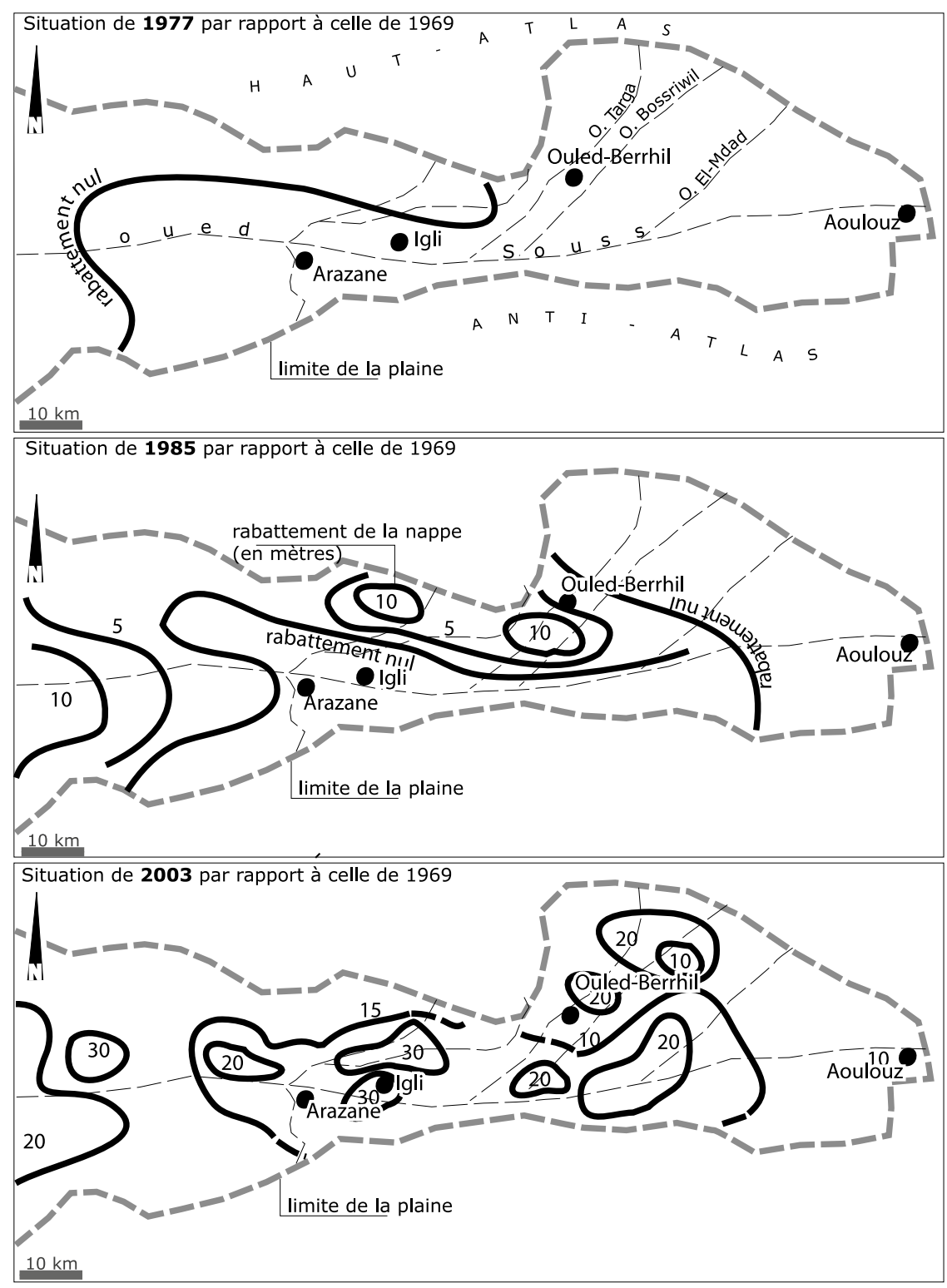

Source : D'après les cartes piézométiques de la plaine du Souss, 1977, 1985, 1996 et 2003, DRH d'Agadir. Voir aussi R. Dijon (1969) et El. Mahdad (2003).

Figure 4 : Aggravation du rabattement de l'aquifère dans le Souss oriental entre 1969 et 2003. (Extrait de Boujnikh, 2008)

Ces trois cartes simplifiées, établies à partir de différentes informations, rendent compte de l'indiscutable rabattement de l'aquifère du Souss amont avec l'installation des périmètres de la colonisation officielle et les progrès de l'agriculture commerciale moderne. Les lâchers du barrage d'Aoulouz ne peuvent avoir que des effets mineurs et très temporaires sur la stabilisation du niveau piézométrique et seulement dans les parties les plus basses de la vallée.

Worsning of ground water level dropping down in the eastern Souss between 1969 and 2003

These three simplified maps established from various information give an account of the indisputable dropping down of the aquifer of the upper Souss with the setting of the areas of an official colonization and the progress of modern commercial agriculture. The releases of the dam of Aoulouz can have only minor and very temporary effects on stabilization of the piezometric level and only in the lowest parts of the Valley. 
fermes ont investi le glacis de rive droite vers le Haut-Atlas, la ruine des systèmes paysans s'est précipitée. Un nombre considérable de khettara(s) alimentait une série de terroirs autour d'Ouled Berrhil (fig. 3). Ces systèmes drainants sont extrêmement sensibles au moindre rabattement des nappes dans lesquelles ils ont été établis (fig. 4). Un abaissement du niveau d'une dizaine de mètres suffit à percher le fond de la galerie au-dessus du toit de la nappe et à la stériliser totalement. Une solution consiste alors à creuser une nouvelle galerie en dessous de la première pour rattraper l'aquifère. L'opération a été réalisée dans la région mais les communautés qui s'étiolent renoncent pour la plupart à ces travaux. Par ailleurs, le surcreusement d'une khettara entraine inévitablement la stérilisation de la partie amont du terroir puisque le débouché à l'air libre est obligatoirement plus bas que l'ancien. De toute façon un nouvel enfoncement de la nappe scelle la mort du drain (photo 6 - planche VII). Les plus pugnaces parmi les paysans tentent de sauver individuellement leurs jardins en creusant des puits à traction animale (puits à dlou ou arghrour). Ces palliatifs sont à leur tour abandonnés parfois après une tentative de motorisation de l'exhaure (Boujnikh, 2008). L'examen systématique du réseau de khettara(s) montre qu'en 2008 la plupart des galeries sont taries (fig. 3 et fig. 5). Les seules qui fonctionnent encore sont celles qui sont établies dans le lit de l'oued Souss et de quelques-uns de ses affluents.

En effet, ces nappes phréatiques - ou ces inféroflux - sont très intéressantes car elles sont présentes dans les alluvions même quand il n'y a plus une goutte en surface, et surtout elles se rechargent après chaque crue. Dans le Souss amont, elles bénéficient par ailleurs des recharges contrôlées réalisées grâce à des lâchers d'eau du barrage d'Aoulouz, mis en service en 1991 avec une capacité de $110 \mathrm{hm}^{3}$. En raison de leur localisation ces drains d'oued présentent une structure

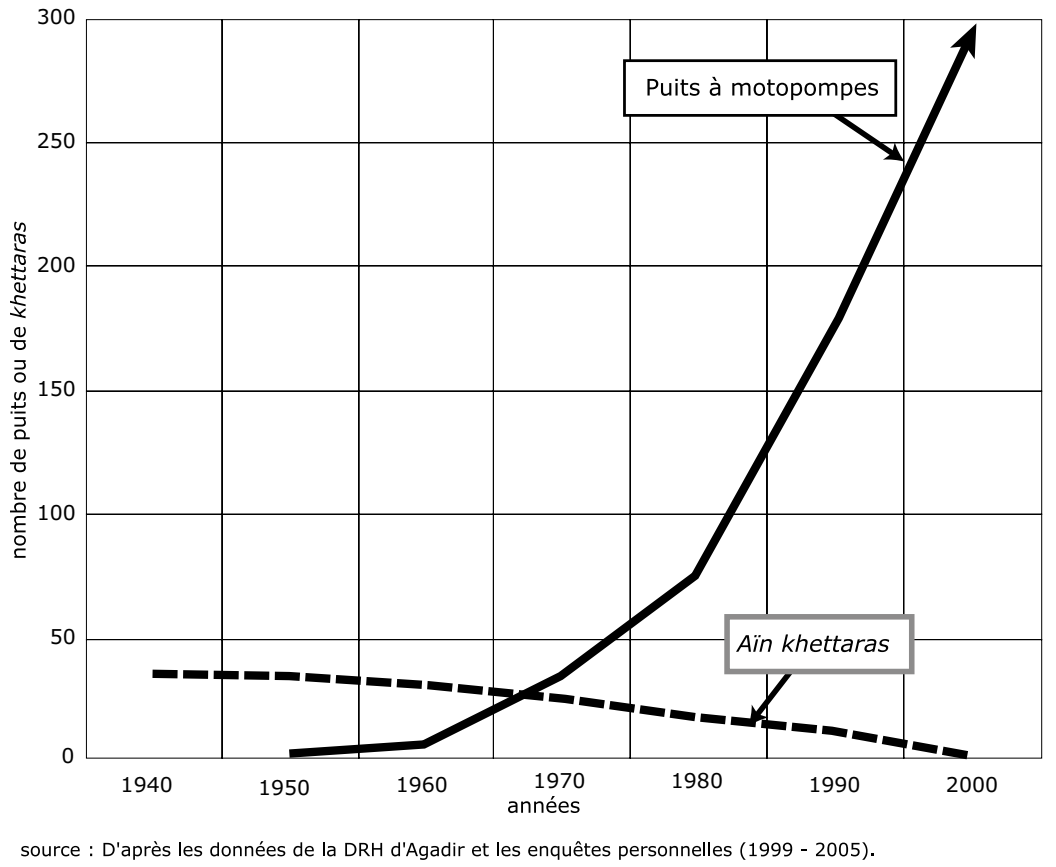

Figure 5 : Évolution du nombre de puits à motopompes et mort des khettara(s) (Extrait de Boujnikh, 2008) Ce diagramme exprime de façon éloquente la corrélation entre le développement de l'agriculture minière du Souss et l'extinction des galeries drainantes (commune de Tamast, Souss amont).

Evolution of the number of wells with power-driven pumps and death of the khettara(s)

This diagram expresses in an eloquent way the correlation between the development of mining agriculture of the Souss and the extinction of the drainage galleries (district of Tamast, upper Souss). 


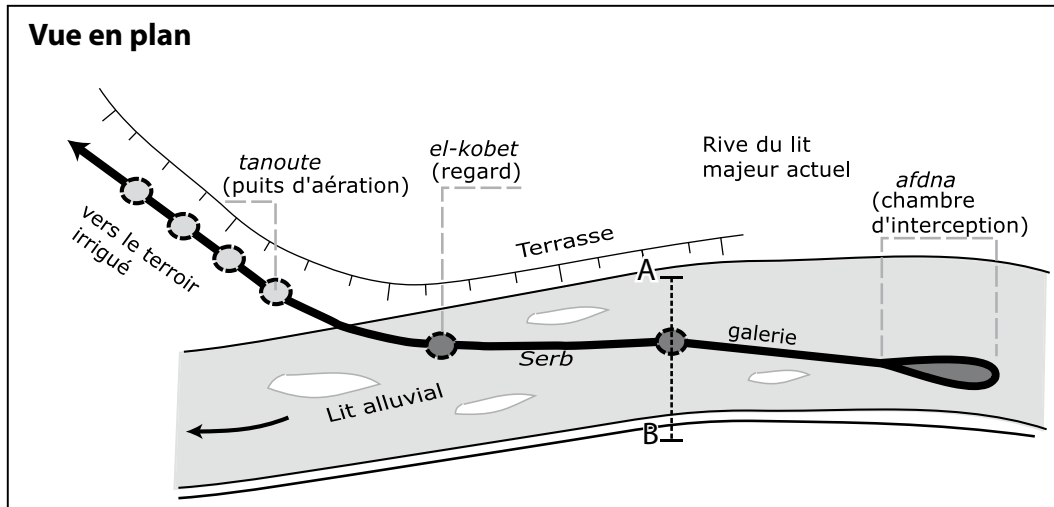

\section{Coupe longitudinale d'un puits regard de visite (el-kobet)}

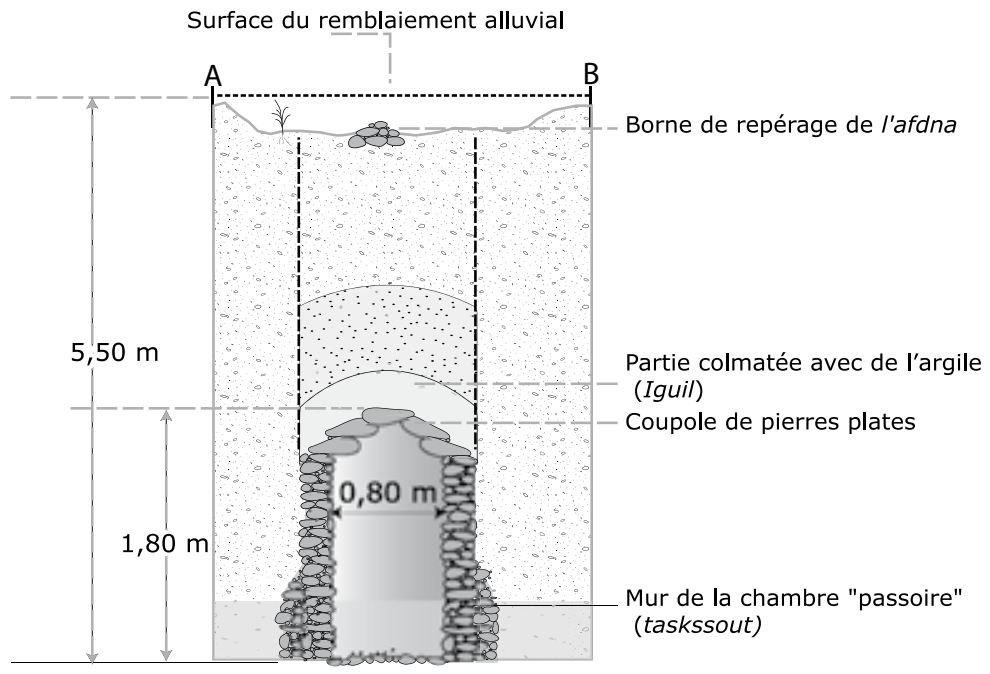

Figure 6 : La Khettara d'oued : un artefact patrimonial en sursis? (Extrait de Boujnikh, 2008)

Ce document réalisé à partir d'observations de terrain explique la structure linéaire et en coupe d'une des rares galeries de captage qui fonctionnent encore dans le Souss. Issu d'une chambre de réception filtrante, la galerie, noyée dans la masse des alluvions du lit de l'oued, est construite avec soin à l'aide de galets et de dalles de pierre. Lorsque la partie proprement drainante $(\mathrm{serb})$ quitte le lit de la rivière pour pénétrer dans la terrasse qui domine le lit mineur, elle devient semblable aux khettara(s) de piémont avec leurs multiples puits repérables en surface (voir photo 6 - planche VII).

The river-bed khettara: a patrimonial artifact under death sentence?

This document realized from field observations explains the linear structure and cross section of the rare galleries of collecting water which still work in the Souss. Stemming from a filtering reception room, the gallery, drowned in the alluvial mass of the bed of the wadi, is built carefully using pebbles and stone slabs. When the properly draining part (serb) leaves the bed of the river to penetrate in the terrace which dominates the minor bed, it becomes like the khettara(s) of piedmont with their multiple locatable wells on the surface (see photo 6 -plate VII). 
particulière et demandent un entretien souvent délicat (Boujnikh, 2008, fig. 6). Ils ont dû jouer un rôle très important dans l'histoire agraire du Souss, non seulement parce qu'ils ont forgé et pérennisé une solide civilisation paysanne fondée sur l'irrigation, mais aussi car ils ont sans doute assuré le succès de la culture de la canne à sucre à l'époque saadienne (xvi ${ }^{\mathrm{e}}$ siècle) (Berthier, 1966). En effet, cette culture tropicale qui a permis d'alimenter en sucre une partie de l'Europe était difficilement concevable sans que des apports en eau soient assurés tout au long de l'été, au moment où la canne était en pleine croissance. Le réseau des grandes seguias charriant l'eau des crues devait très probablement être alimenté en été par un réseau de khettara(s) d'oued (Humbert, 2002).

Mais aujourd'hui, les quelques khettara(s) d'oued qui fonctionnent encore sont bien incapables de sauver les vieux terroirs qui sont d'ailleurs, pour la plupart, hors d'atteinte de leurs seguia(s). La métamorphose des paysages est spectaculaire. Les terroirs aux parcellaires anarchiques d'antan disparaissent sous le double effet de leur stérilisation par l'enfoncement de la nappe et de la poussée conquérante des grandes fermes à motopompes. Ce phénomène est particulièrement net dans le secteur des Ouled Berrhil précisément (fig. 7), où la dernière vague de l'agriculture minière contemporaine est si agressive. Les terroirs paysans meurent mais il arrive aussi, déjà, que les motopompes s'essoufflent à poursuivre les nappes et qu'il faille alors renoncer à arroser les orangers en acceptant de les voir sécher sur pied (photo 7 - planche VIII).

\section{Conclusion}

Nous observons donc aujourd'hui la ruine des systèmes anciens dont il ne restera bientôt plus que les vestiges qui peu à peu vont s'évanouir. Rapidement vont s'effacer les alignements de puits des khettara(s), tout comme vont se combler les seguia(s) et les bassins d'accumulation. Une vieille civilisation de l'eau est en train de disparaître, tuée par une agriculture commerciale intensive et capitaliste qui se livre à une véritable économie minière en prélevant chaque année dans le sous-sol quelque $650 \mathrm{hm}^{3}$ d'eau, c'est-à-dire bien plus que les apports annuels d'environ $350 \mathrm{hm}^{3}$ (Baroud, El Arabi, 2005). À défaut d'un retour improbable à un équilibre raisonnable, sa mort est annoncée, comme est morte, pour d'autres raisons l'agriculture « capitaliste » de la culture saadienne de la canne à sucre. C'est probablement sur les ruines de cette dernière que s'est établie une partie des terroirs irrigués des rives de l'oued Souss, en réutilisant partiellement des éléments des anciens réseaux et notamment ceux qui extrayaient du fleuve les eaux enfouies dans les alluvions. La naissance - ou renaissance - d'une civilisation hydraulique paysanne sur les ruines d'un grand système d'état peut-t-elle entretenir l'espoir d'un rétablissement futur après le déclin du système prédateur actuel? Comparaison n'est pas raison. L'agriculture sucrière ne prélevait que dans des aquifères superficiels ou à moyenne profondeur et ceux-ci s'autorégulaient. Les nappes fossiles profondes n'étaient pas sollicitées. La mort de l'industrie sucrière a pu déséquilibrer la situation de certains réseaux confrontés à une remontée générale des niveaux piézométriques, en particulier dans les galeries de captage, et les communautés paysannes ont probablement dû faire un effort de réorganisation en récupérant une partie seulement de l'héritage. Entre les deux types d'économies qui se sont succédés, il n'y a pas eu de véritable rupture technologique. L'état saadien avait certes les moyens de faire les choses en grand, mais les techniques qu'il mettait en œuvre pour récupérer de l'eau n'étaient pas foncièrement différentes de celles qu'ont employées ensuite, jusqu'au xx ${ }^{\mathrm{e}}$ siècle, les communautés de fellah(s). L'épuisement systématique des gisements aquifères profonds par les moyens mécaniques actuels pourrait bien, au contraire, déboucher sur la désertion d'une forme d'agriculture qui ne trouverait plus ici son intérêt, après avoir désorganisé totalement une société paysanne dispersée au vent de la mondialisation. Mais le pire n'est jamais certain et la géographie n’est pas une science prophétique. 


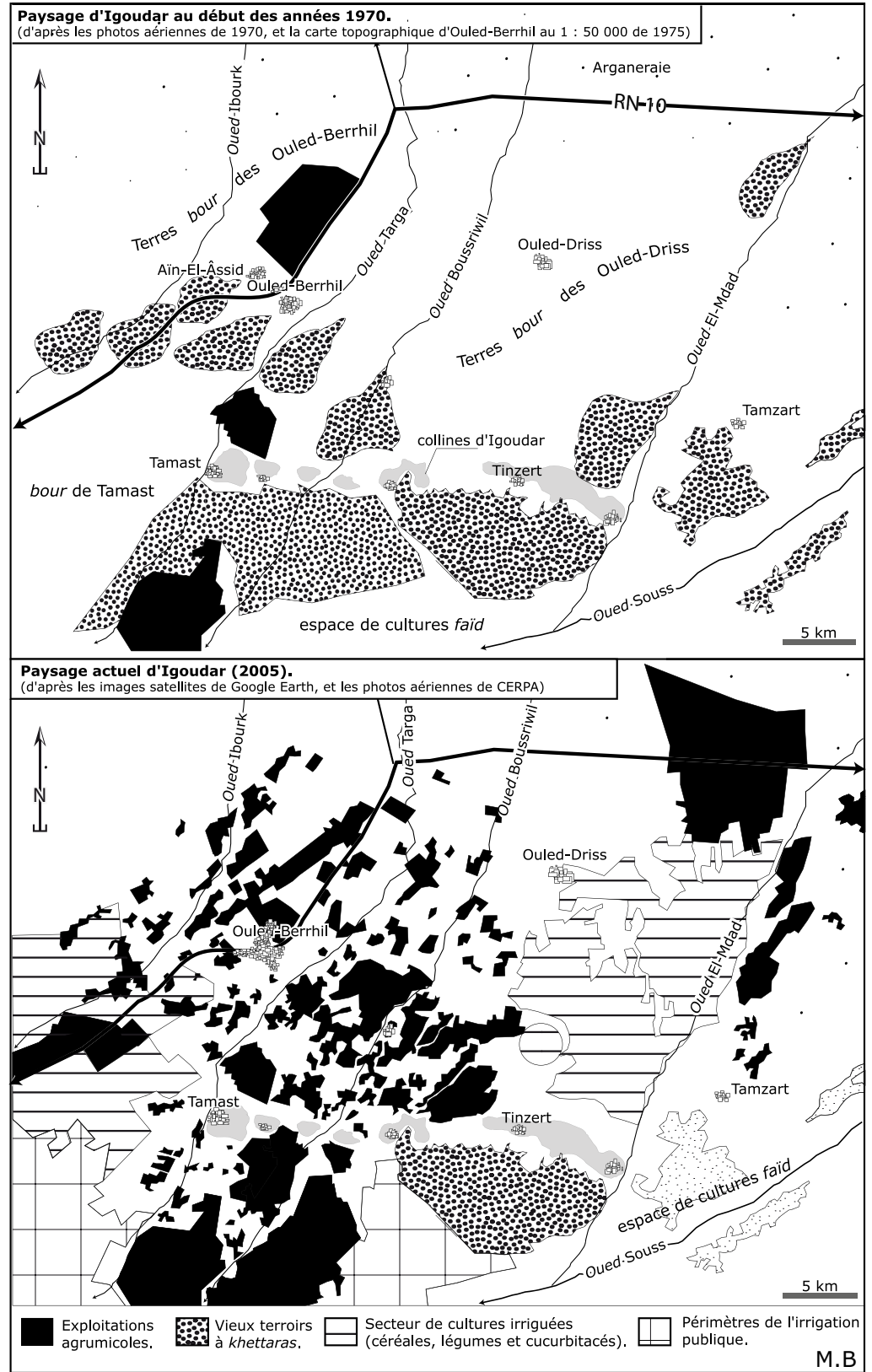

Figure 7 : Mutation des paysages agraires sous l'effet de l'agriculture minière moderne dans la région des Ouled Berrhil (Extrait de Boujnikh, 2008)

Les terroirs traditionnels ont fondu comme neige au soleil alors que proliféraient les fermes d'agrumes et les serres. Le soutirage dans l'aquifère est aggravé encore par la proximité du périmètre de la « réforme agraire » (colonisation officielle).

Transformation of the agrarian landscapes under effects of modern mining agriculture in the Ouled Berrhil region

The traditional fields melted away like the snow whereas the citrus fruits farms and the greenhouses proliferated. Pumping in the aquifer is still worsened by the proximity of the "land reform" area (official colonization). 
Mohamed Boujnikh, André Humbert

\section{Remerciements}

Les recherches dans le Souss et les observations aériennes qui ont produit les photos utilisées n'ont été possibles que grâce à l'aide du Comité Mixte Interuniversitaire Franco-Marocain.

\section{Bibliographie}

Balland D. (dir.), 1992. Les eaux cachées : études géographiques sur les galeries drainantes souterraines, Paris, Lavoisier, Publication $n^{\circ} 19$.

Baroud A., El Arabi E., 2005. Les ressources en eau et les équipements hydroagricoles dans la zone du Souss-Massa, dans Bouchelkha M. (dir.), L'espace rural dans le Souss. Héritages et changements, Actes du colloque d'Agadir (15-16 mars 1996), p. 85-94.

Berriane M., 2002. Les angles dynamiques : le Souss-Massa et ses prolongements, dans Troin J.-F. (dir), Maroc. Régions, pays, territoires, Paris, Maisonneuve et Larose, p. 295-324

Berthier P., 1966. Un épisode le l'histoire de la canne à sucre : les anciennes sucreries du Maroc et leurs réseaux hydrauliques, Étude archéologique et d'histoire économique, Paris, CNRS, 2 vol., 350 p.

Boujnikh M., 2008. Évolution des paysages irrigués dans le Souss oriental. De la khettara à la motopompe; des terroirs faïd aux grands périmètres irrigués (Le cas des Ouled Berrhil, province de Taroudant), Thèse de doctorat en géographie, université Nancy 2, 552 p.

Déspois J., Raynal R., 1967. Géographie de l'Afrique du Nord-Ouest, Paris, Payot, 570 p.

Dijon R., 1969. Étude hydrogéologique et inventaire des ressources en eau de la vallée du Souss, Thèse de doctorat-ès-sciences, Université de Montpellier, 300 p.

Galland F., 2008. L'eau. Géopolitique, enjeux, stratégies, Paris, CNRS, 192 p.

Hnaka A., 1995. Taroudant et Ouled Teima, bipôle urbain du Souss (Maroc), Agadir, université Ibnou Zohr, Série Thèses et mémoire-4, $340 \mathrm{p}$.

Humbert A., 2002. Archéologie d'un système hydraulique. L'ancienne sucrerie d'Ouled Messaoud et le système hydraulique de la plaine du Souss, dans Aïт Hssaine A., Humbert A. (dir.), Eau, environnement et histoire des paysages dans le Sous, Actes du colloque de Taroudant, novembre 2000, Agadir/Nancy, université d'Agadir/université de Nancy 2/Comité Mixte Interuniversitaire Franco-Marocain, CD-Rom, 17 écrans.

Humbert A., 2006. Comment mesure-t-on l'eau d'irrigation dans les communautés traditionnelles du Maroc méridional ?, dans Cressier P. (dir.), La maîtrise de l'eau en al-Andalus. Paysages, pratiques et techniques, Madrid, Collection de la Casa de Velázquez, 2006, p. 313-325.

Lugan B., 2000. Histoire du Maroc des origines à nos jours, Paris, Périn-Critérion, 367 p.

Margat J., 2008. L'eau des Méditerranéens, Paris, L'Harmattan, 288 p.

Mutin G., 2000. L'eau dans le monde arabe. Enjeux et conflits, Paris, Ellipses, coll. "Carrefours de géographie », 156 p.

Martin P., Jover H., Le Coz J., Maurer G., Noin D., 1970. Géographie du Maroc, Paris, Hatier, 256 p.

Pérennès J.-J., 1993. L'eau et les hommes au Maghreb. Contribution à une politique de l'eau au Maghreb, Karthala, 646 p.

Popp (H.), 1984. Un «man made hazard » : le surpompage dans la vallée du Souss. Aspects socio-géographiques d'une exploitation excessive des eaux souterraines, Revue Géographique du Maroc, $\mathrm{n}^{\circ}$ 7, nouvelle série, p. 35-49.

Popp H., 1986. L'agriculture irriguées dans la vallée du Souss (Maroc), Formes et conflits d'utilisation de l'eau, Méditerranée, n 4, p. 33-47.

Troin J.-F. (dir.), 1985. Le Maghreb. Hommes et espaces, Paris, Armand Colin, coll. « U », 360 p.

Cet article a été reçu le 19 février 2009 et définitivement accepté le 6 aô̂t 2009. 
planche II (Mohamed BOUJNIKH, André HUMBERT - L'eau dans le bassin du Souss...)

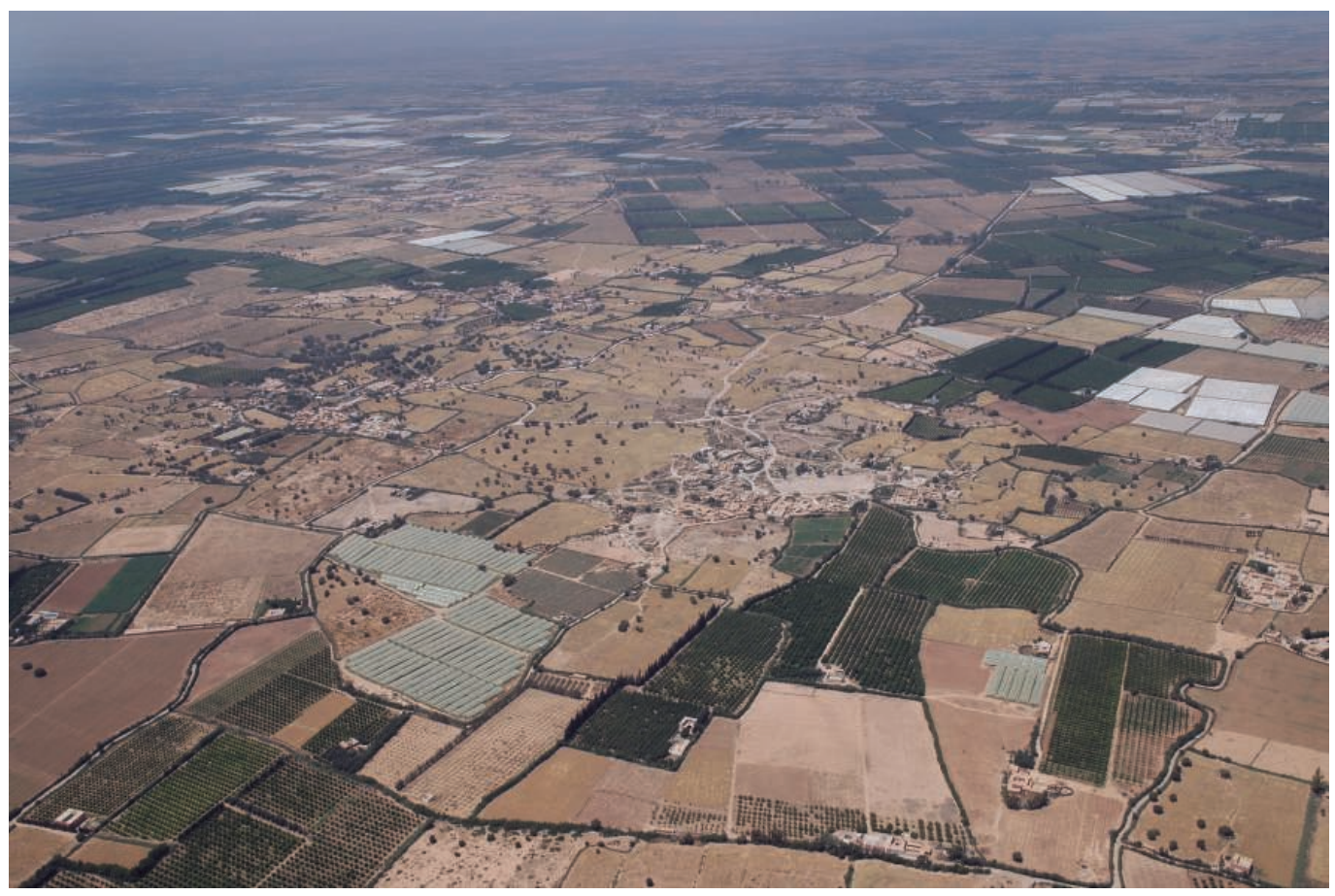

Photo 1 : Paysage mixte de la partie centrale de la plaine du Souss (région d'Ouled Teima (A. Humbert et C. Renard/ CMIFM, avril 2005)

Autour d'un cercle central partiellement occupé par des douars à la structure anarchique, une vaste couronne est occupée majoritairement par des parcelles géométriques d'orangers et de serres. L'agriculture intensive et productive est dans cette périphérie alors que le terroir paysan semble aujourd'hui stérilisé ou réduit à une culture bour de céréales déjà mûres en cette fin du mois d'avril.

Mixed landscape in the central part of the Souss Valley (Ouled Teima region, April 2005)

Around a central circle partially occupied by douar(s) with the anarchistic structure, a vast crown is mainly occupied by geometrical plots of orange trees and greenhouses. The intensive and productive agriculture is in this periphery whereas the peasants' fields seems sterilized today or reduced to a bour cultivation of cereals already ripe in this end of April. 
planche III (Mohamed BOUJNIKH, André HUMBERT - L'eau dans le bassin du Souss...)

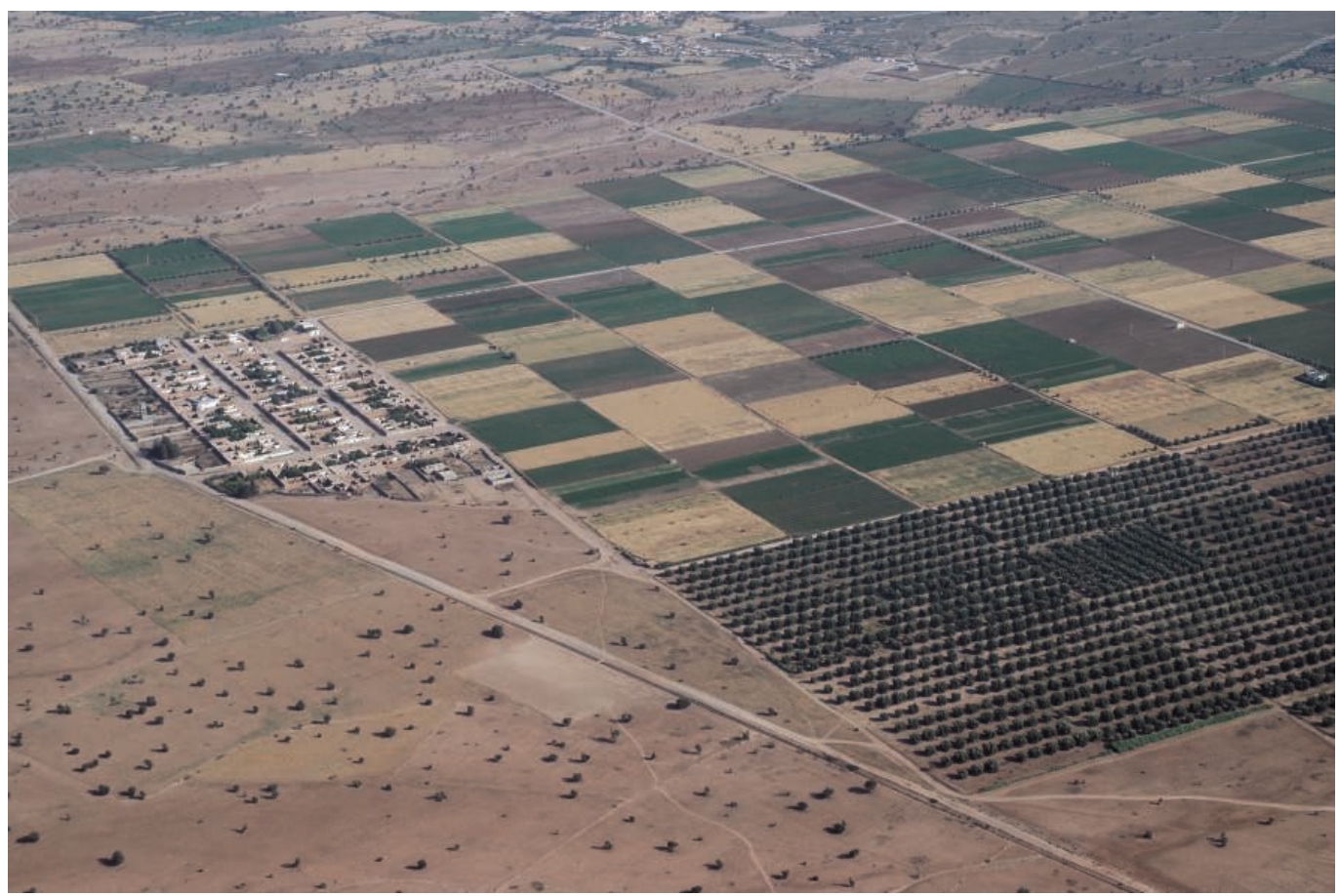

Photo 2 : Périmètre de colonisation de la réforme agraire dans la région de Taroudant (commune de Freija) (photo A. Humbert et C. Renard/CMIFM, avril 2005)

Initié dans les années 1970, le programme de création de périmètres de la réforme agraire a donné naissance à ce paysage d'une grande rigueur géométrique. Les lots d'environ 5 ha étaient, en principe soumis à une répartition rigoureuse des cultures (céréales, légumes, fourrage...) sans cultures fruitières telles que celle des agrumes. Les parcelles d'oliviers autorisées ( 1 ha) étaient toutes réunies dans le même secteur du périmètre. Le village « de colonisation » présente le même systématisme que le parcellaire. L'alimentation en eau de ces périmètres est assuré par de puissantes stations de pompage qui contribuent aussi, bien évidemment, au rabattement du niveau de l'aquifère.

Area of the agrarian reform colonization in the Taroudant region(Freija district) (April 2005)

Get under way in the 1970s, the programme of creation of irrigation areas of the land reform gave rise to this landscape of a great geometrical rigour. The (plots) of approximately 5 hectares, were subjected in theory to a rigorous distribution of the crops (cereals, vegetables, fodder...) without fruit-bearing trees such as citrus trees. The plots of olive-trees authorized (1 ha) were all joined together in the same sector. The village "of colonization" presents the same regular scheme that the fields organization. The water supply of these areas is obtained by powerful stations of pumping which also contribute, obviously, to the dropping down of the level of the aquifer. 


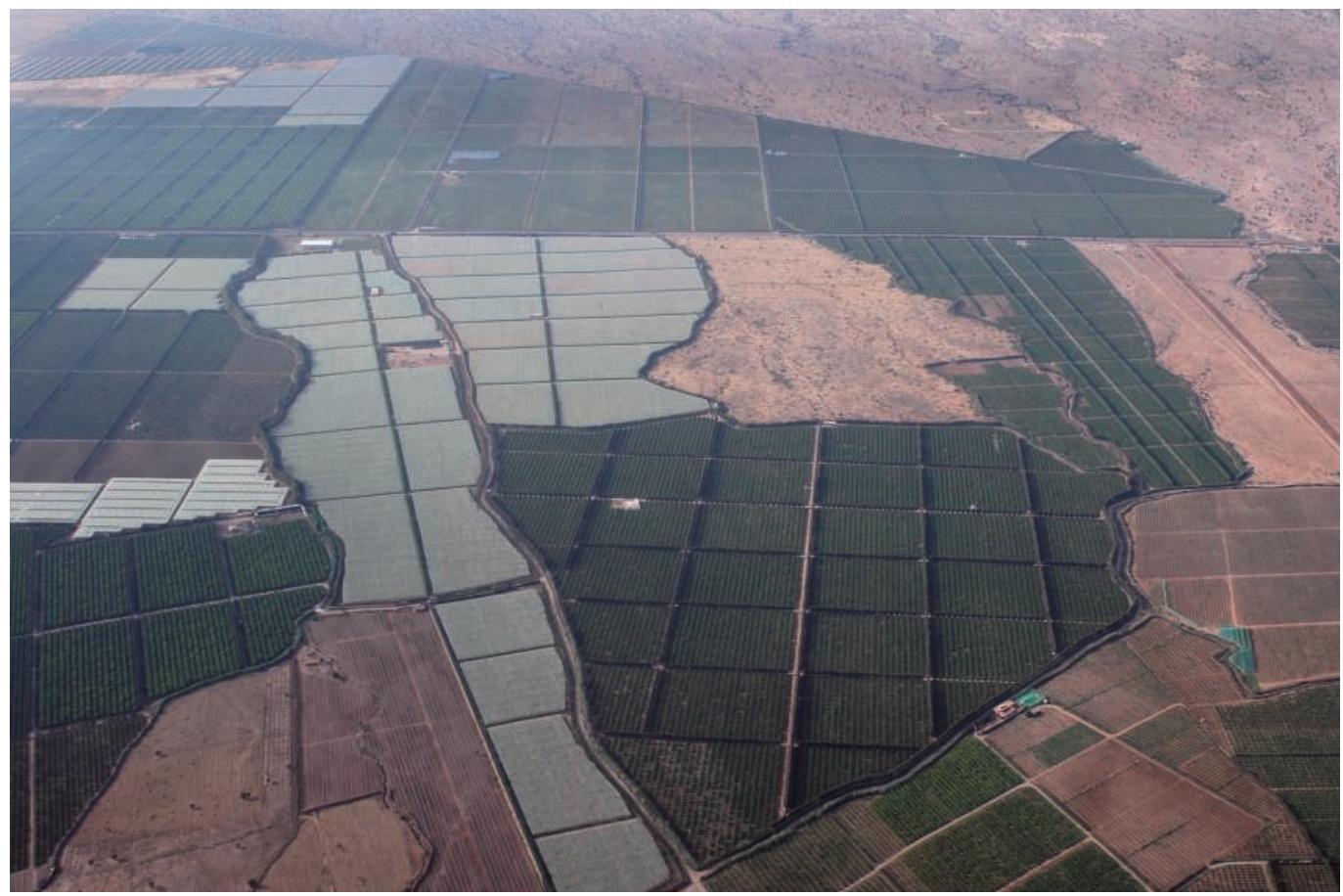

Photo 3 : Grande exploitation de cultures mixtes chez les Ouled Berrhil (Souss amont) (photo A. Humbert et C. Renard/CMIFM, avril 2006)

Le glacis de piémont du Haut-Atlas occupé par un peuplement clair d'arganiers servait traditionnellement de terrain de parcours et de terroir bour pour une culture aléatoire d'orge. Il est aujourd'hui très largement investi par des fermes parfois de très grande taille dont les propriétaires ont foré de multiples puits pour cultiver des agrumes mais aussi pour y installer d'immenses serres, ce qui leur permet de rentabiliser rapidement leurs investissements. On peut constater que les vergers d'agrumes sont d'âges très variés, ce qui traduit, à la fois le dynamisme de l'exploitation et l'avidité hydraulique. La géométrie rigoureuse est de règle sauf à adapter les blocs de culture et la structure des serres aux sinuosités des ravinements [talate(s)] qui griffent la surface du glacis.

Large farm of mixed crops in Ouled Berrhil area (upper Souss Valley, April 2006)

The glacis of piedmont of the High-Atlas occupied by a sparse planting of argan-trees was used traditionally as pasture and for bour cultivation of barley. It is very largely occupied today by farms, sometimes of very big size, whose owners drilled many wells to cultivate citrus fruits but also to install immense greenhouses there, which enables them to make profitable their investments quickly. One can to note that the citrus fruits orchards aren't all the same age which express, at the same time, the dynamism of the exploitation and hydraulic greed. The rigorous geometry is the rule except to adapt the blocks of cultivation and the structure of the greenhouses to the winding gullyings [talate(s)] who scratch the surface of the glacis. 
planche V (Mohamed BOUJNIKH, André HUMBERT - L'eau dans le bassin du Souss...)

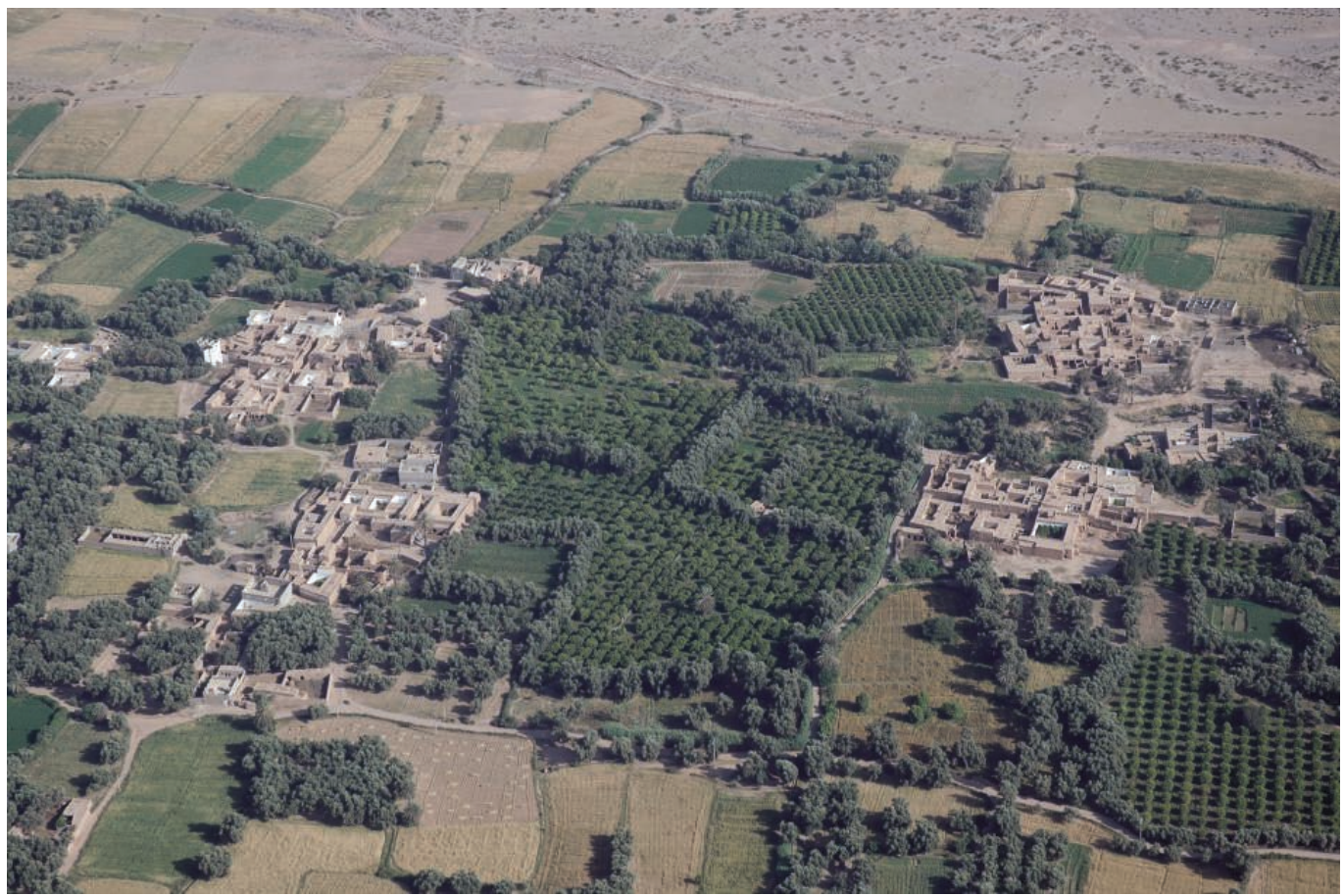

Photo 4 : Paysage traditionnel modifié dans le Souss amont (photo A. Humbert et C. Renard/CMIFM, avril 2005)

Tout à fait à l'amont de la dépression, les paysages des bords de l'oued Souss ont gardé l'essentiel de leurs caractères et ils continuent souvent à être irrigués grâce à l'eau des khettara(s) entretenues dans le lit du fleuve. On remarquera ici, cependant, que les cultures commerciales, celles des agrumes en particulier, font leur apparition en s'installant dans les mailles du parcellaire ancien au dessin souligné par des rangées de vieux oliviers.

Modified traditional landscape in the upper Souss valley (April 2005)

In the uppermost part of the depression, the landscapes of the edges of the wadi Souss kept their main characteristics and they often continue to be irrigated thanks to the water of the khettara(s) maintained in the bed of the river. It will be noticed here, however, that the commercial crops, those of citrus fruits in particular, make their appearance while settling in the meshes of compartmentalized old fields with the drawing underlined by lines of old olive-trees. 


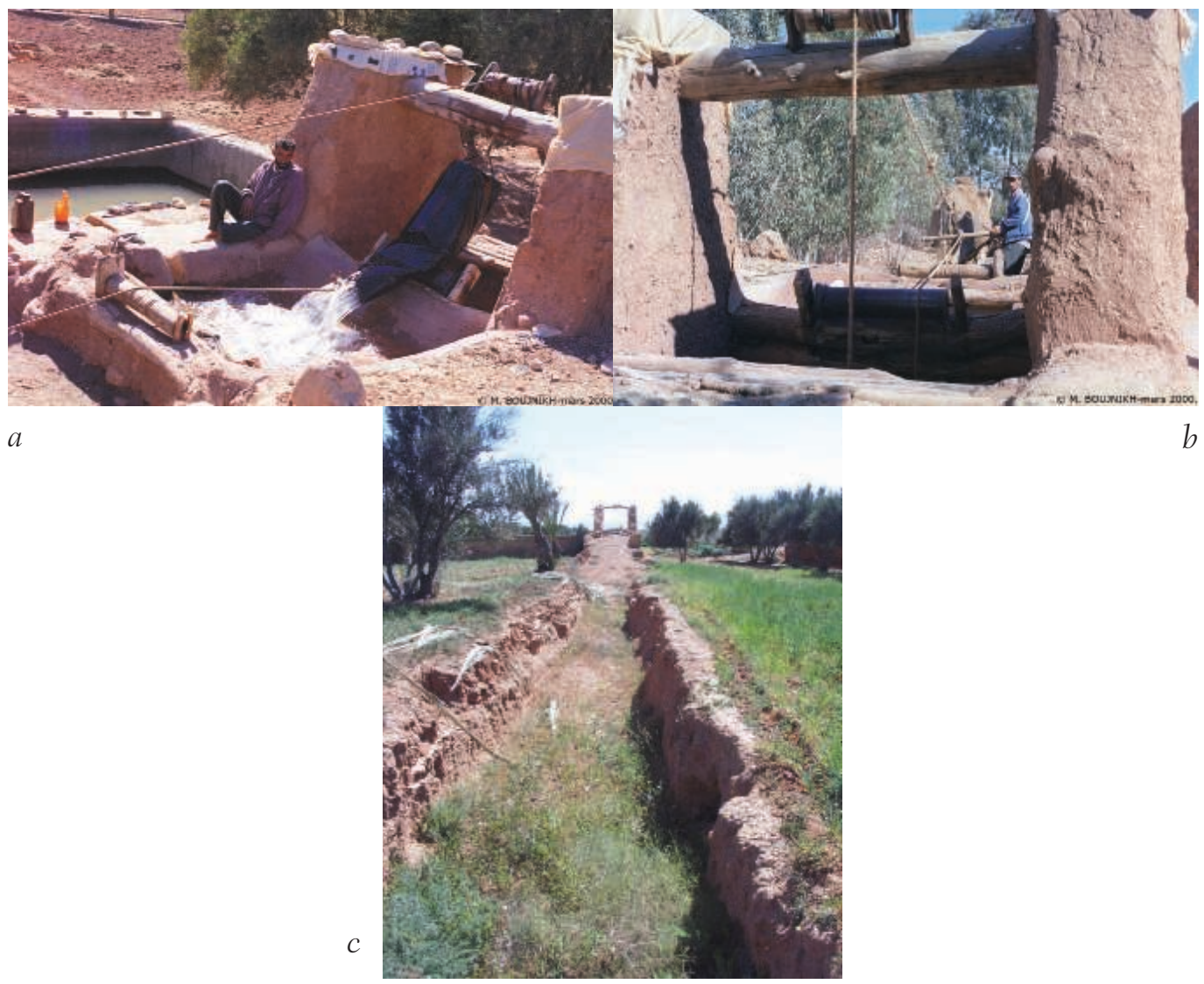

Photos 5a, b et c : L'arghrour, vieil artefact hydraulique en voie d'extinction

Presque partout dans la vallée du Souss, le rabattement des aquifères a asséché les puits familiaux que les fellah(s) se sont fatigués d'approfondir.

a) : Quelques arghrour(s) fonctionnent encore. Le principe de ce puits dit aussi « à dlou », c'est-à-dire «à outre », est fondé sur les allers-retours d'un animal qui descend et remonte la poche suspendue par deux cordes dont l'une, à l'arrivée, libère l'orifice de vidange. Le dlou, ici en caoutchouc, était autrefois le plus souvent une peau de vache. L'eau déversée dans un petit bassin est conduite dans le grand bassin de stockage (sur la gauche) d'où elle sera répartie dans les différents casiers du jardin.

b) : Le dromadaire convient particulièrement bien pour remonter les grosses poches d'eau du puits à dlou; il est guidé par un fellah sur la piste de halage; celui-ci surveille, en même temps le glissement des cordes sur les poulies en bois d'olivier ou d'arganier.

c) (A. Humbert et C. Renard, février 2003) : Arghrour abandonné dans la plaine des Chtouka. Le puits dresse encore ses deux piliers reliés par la poutre qui supportait le dlou; la pente de la piste facilitait le travail de l'animal lors de la remontée de l'outre. Parfois ces vieux puits servent de point de départ pour un forage équipé d'une motopompe.

The arghrour well, old hydraulic artefact on the way to vanishing

Almost everywhere in the Souss Valley, the dropping down of the aquifers dried out the family wells that the fellah(s) got tired to deepen.

a: Some arghrour(s) still work. The principle of this well also called "dlou well", i.e. "with water skin", is founded on the "go and return" of an animal which descends and pull again the pocket suspended by two cords of which one, on arrival, releases the opening of the outlet. The dlou, here out of rubber, was formerly a skin of cow, generally. The water poured in a small basin is led in the large basin of storage (on the left) from where it will be distributed in the various compartments of the garden.

b: The dromedary is particularly well appropriate to pull the large pockets of water out of the well; it is guided by one fellah on the track of hauling; this one supervises, at the same time, the slip of the cords on the pulleys made of wooden of olive-tree or argan-tree.

$c$ : Disused arghrour in the plain of Chtouka. The well still erect its two pillars connected by the beam which supported the dlou; the slope of the track facilitated the work of the animal at the time of pulling off the water skin. Sometimes these old wells are used as starting point for a drilling equipped with a motor-driven pump. 


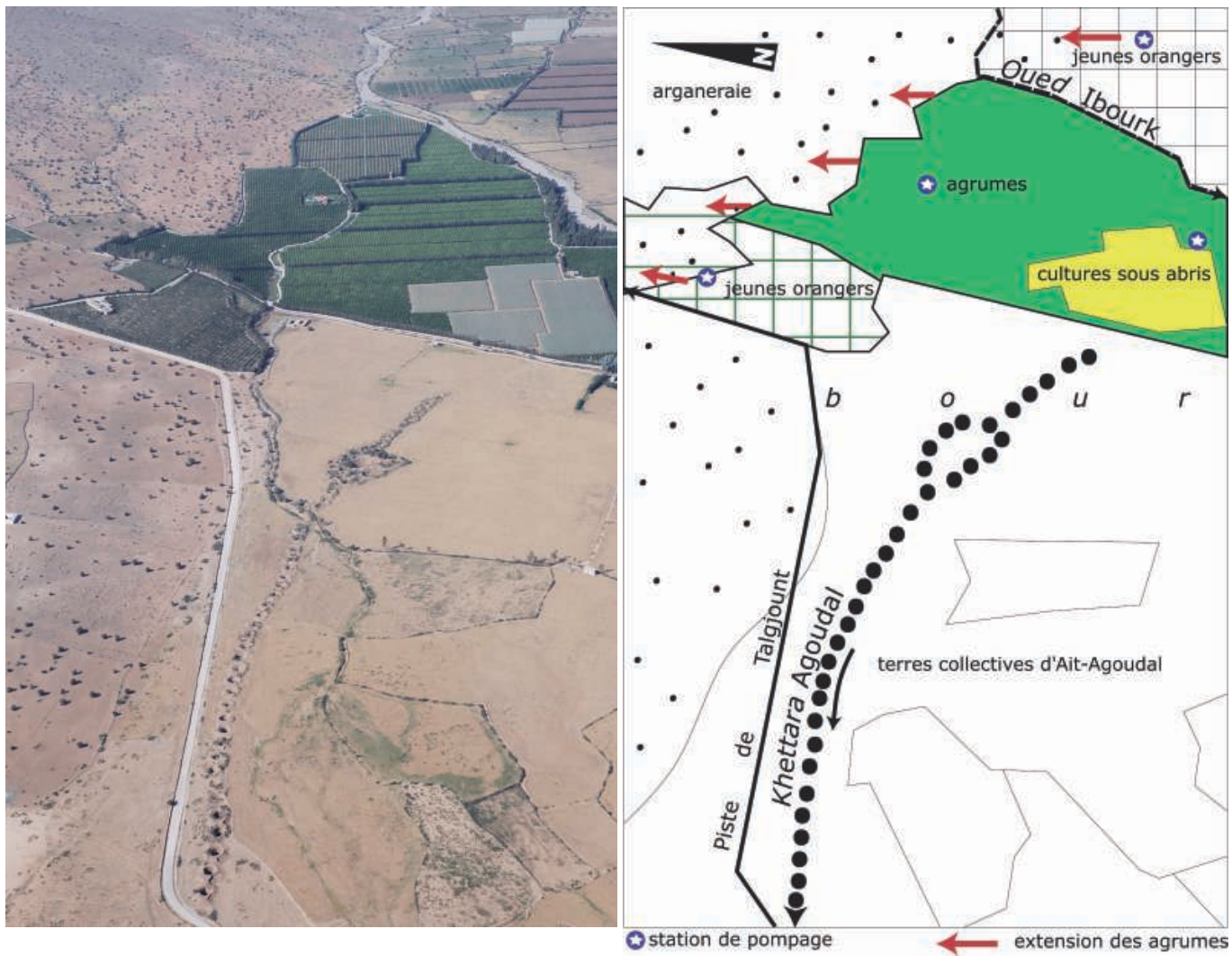

Photo 6 : Khettara stérilisée par le pompage de l'aquifère dans les fermes de culture commerciales. (Photo A. Humbert et C. Renard/CMIFM, avril 2005)

Cette photographie aérienne oblique résume parfaitement la situation des artefacts traditionnels d'irrigation dans la plaine du Souss orientale. La khettara d'Agoudal desservait un terroir de la partie inférieure du glacis de rive droite. La grande ferme d'agrumes et de cultures maraîchères installée à l'amont a rapidement provoqué un rabattement de la nappe dans «l'entonnoir» de pompage. Un ultime effort pour tenter de revitaliser le drain est exprimé par les quelques puits creusés à l'amont de la tête « en grappe » de la khettara. Espoir illusoire, évidemment. La khettara est bien morte, définitivement.

Dried khettara by pumping of commercial crops farms (april 2005)

This oblique air photography summarizes perfectly the situation of the traditional artifacts of irrigation in the eastern plain of the Souss. The khettara of Agoudal served fields of the lower part of the right bank glacis. The large farm of citrus fruits and market gardening installed in the upper valley quickly caused a lowering of the water level in "the funnel" of pumping. An ultimate effort to try to revitalize the drain is expressed by the few wells dug above the head "in bunch" of the khettara. Illusory hope, obviously. The khettara really died, definitively. 
planche VIII (Mohamed BOUJNIKH, André HUMBERT - L'eau dans le bassin du Souss...)

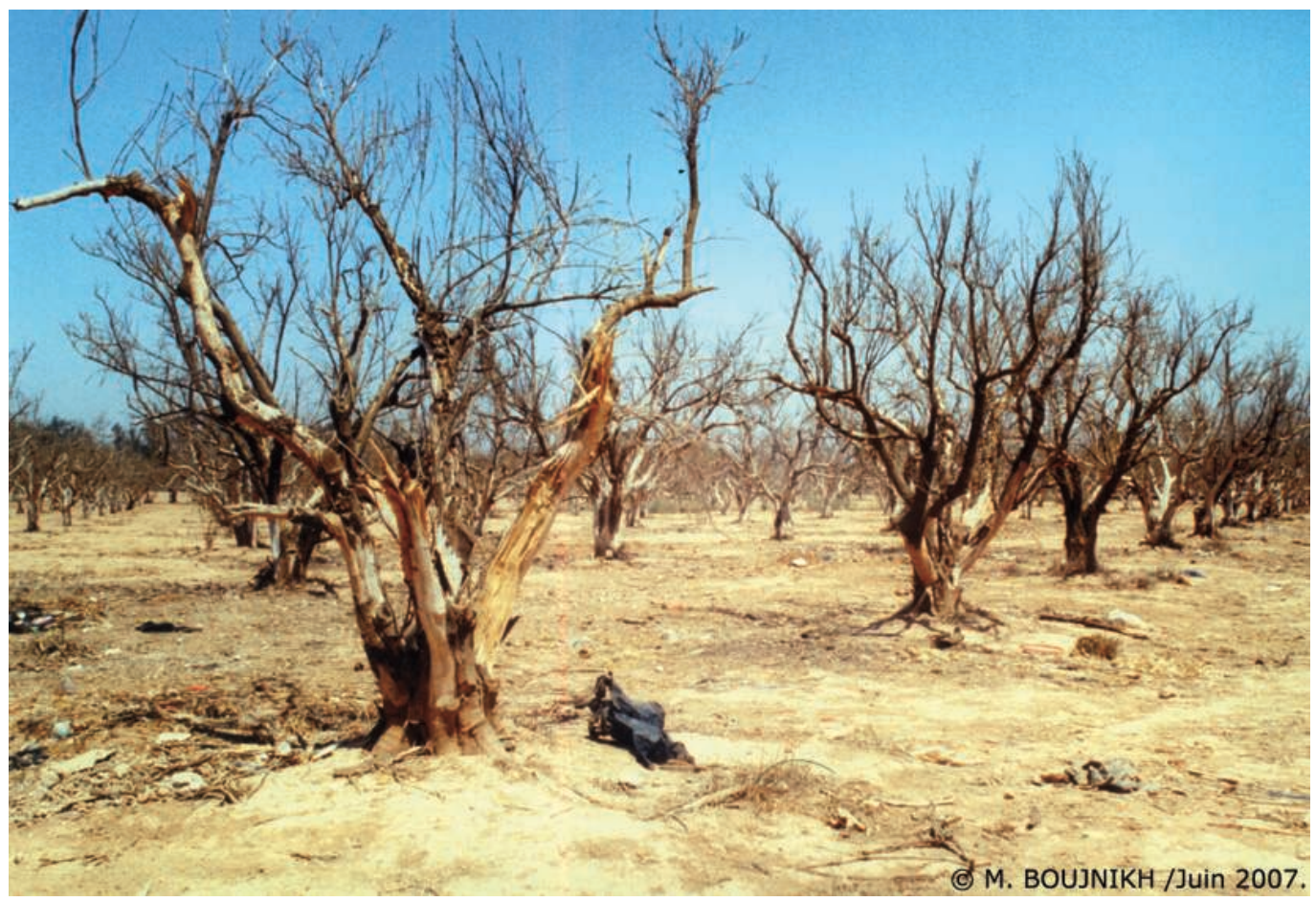

Photo 7 : Orangeraie morte dans une grande exploitation de la région d'Ouled Teima (commune de Sebt-el-Guerdane)

Ce spectacle sinistre peut être observé dans le secteur pionnier de la culture des agrumes, autour d'Ouled Teima. Cette parcelle d'orangers fait partie d'une grande exploitation dont le propriétaire a renoncé à « poursuivre » l'aquifère parce qu'il estimait qu'il avait atteint, vers le bas, le seuil de rentabilité de cette agriculture minière.

2007)

Dead orange grove in a large farm of the Ouled Teima region (Sebt-el-Guerdane district) (June

This sinister spectacle can be observed in the pioneer sector of the citrus fruits cultivation, around Ouled Teima. This plot of orange trees belongs to a large farm whose owner gave up to seek after the aquifer because he estimated that he had reached, to the bottom, the break-even point of this mining agriculture. 